\title{
Estudo comparativo in vitro da resistência ao cisalhamento da colagem e do índice de remanes- cente adesivo entre os compósitos Concise e Fill Magic
}

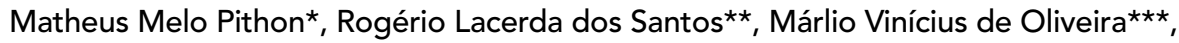 \\ Antônio Carlos de Oliveira Ruellas ${ }^{\star \star \star \star}$
}

\begin{abstract}
Resumo
Objetivo: comparar a resistência ao cisalhamento da colagem e o Índice de Remanescente Adesivo (IRA) entre os compósitos Concise e Fill Magic Orthodontic. Metodologia: a amostra consistiu de 24 incisivos inferiores permanentes bovinos divididos em dois grupos. No primeiro grupo foram colados 12 braquetes com o compósito Concise e no segundo grupo 12 com o compósito Fill Magic Orthodontic. Foi feito o ensaio de cisalhamento e a avaliação do IRA de toda a amostra. Resultado e Conclusão: não foram encontradas diferenças estatísticas significantes entre as colagens, nos dois itens avaliados.
\end{abstract}

Palavras-chave: Colagem. Resistência ao cisalhamento. Descolagem.

\section{INTRODUÇÃO}

Com a introdução da técnica de condicionamento ácido, os procedimentos de montagem do aparelho ficaram mais práticos. Com isso surgiram vários métodos de colagem, sendo usados diferentes materiais. A partir da década de 70, a Ortodontia passou por uma revolução no que diz respeito aos materiais utilizados para fixação de acessórios aos dentes ${ }^{5,19}$.

O primeiro artigo de que se tem referência sobre a colagem de braquetes diretamente sobre a superfície dentária foi descrito por Sadler em
$1958^{20}$, entretanto, na década de 70, assistiu-se a substituição gradual da bandagem pela colagem, exceto para os molares ${ }^{12}$.

Atualmente, vários materiais de colagem têm sido usados, entre eles os compósitos, que possuem boa resistência, dureza e estabilidade dimensional, ${ }^{11}$ embora deixem a desejar quanto à viscosidade e tempo de trabalho ${ }^{6,19}$.

Um material deve ter resistência ao cisalhamento da colagem em torno de 60 a $80 \mathrm{KgF} / \mathrm{cm}^{2}$ para suprir as necessidades clínicas ${ }^{18}$. O compósito Concise, usado em vários trabalhos, promove

\footnotetext{
* Especialista em Ortodontia pela Universidade Federal de Alfenas-UNIFAL. Mestrando em Ortodontia pela Universidade Federal do Rio de JaneiroUFRJ.

** Especialista em Ortodontia pela Universidade Federal de Alfenas-UNIFAL. Mestrando em Ortodontia pela Universidade Federal do Rio de JaneiroUFRJ.

*** Especialista em Ortodontia pela Universidade Federal de Alfenas-UNIFAL.

**** Doutor em Ortodontia pela Universidade Federal do Rio de Janeiro-UFRJ. Professor adjunto de Ortodontia da Universidade Federal do Rio de Janeiro- UFRJ. Professor do curso de especialização em Ortodontia da Universidade Federal de Alfenas-UNIFAL.
} 
adequada resistência dos acessórios à superfície do esmalte. Este compósito foi comparado com cimentos de ionômero de vidro ${ }^{7,8,14,16,22}$, adesivos ácidos $^{1,23}$ e outros compósitos ${ }^{4,13}$, apresentando valores superiores de resistência da colagem ao cisalhamento.

Entre os compósitos atuais que estão surgindo no mercado, cita-se em especial o Fill Magic Orthodontic que ainda é um compósito pouco testado e estudado. Porém na literatura existe uma infinidade de trabalhos, comparando compósitos entre si, e também compósitos com cimentos ionoméricos. A literatura e a prática clínica mostram que é possivel obter uma colagem satisfatória com vários materiais, cada qual com a sua técnica. Entretanto, como a ciência é fascinante e infinita, sempre se deve estar pesquisando e tentando descobrir as vantagens, desvantagens, usos e limitações dos vários produtos odontológicos para se saber quais materiais podem ser usados, a fim de obter um resultado com mais benefícios ao paciente.

Assim sendo, o objetivo do presente trabalho foi comparar os compósitos Concise e Fill Magic Orthodontic quanto aos requisitos resistência ao cisalhamento e IRA na superfície dentária após a remoção do braquete. Como trabalhos anteriores já demonstraram a aplicabilidade clínica do Concise, por meio desta comparação será verificado se o Fill Magic também pode ser utilizado clinicamente com igual sucesso.

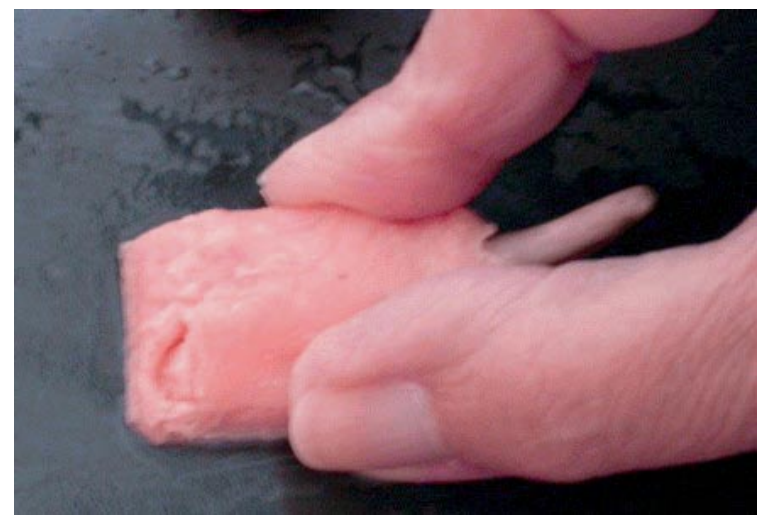

FIGURA 1 - Superfície vestibular do dente sendo lixada.

\section{MATERIAL E MÉTODOS}

Foram utilizados 24 incisivos inferiores permanentes bovinos, armazenados em solução de formol a $10 \%$ e estocados em geladeira a 6 graus centígrados. A coroa destes dentes e pequena parte da raiz foram incluídas em resina acrílica autopolimerizável, com o apoio de uma placa de vidro que serviu como planificador da face vestibular. Após a presa da resina, a face vestibular dos dentes foi lixada com lixas d'água (Fig. 1) até que se conseguisse uma superfície de esmalte uniforme com diâmetro de aproximadamente $5 \mathrm{~mm}$. Em seguida cada dente foi incluído em tubo PVC de maneira centralizada com gesso pedra tipo IV, ficando

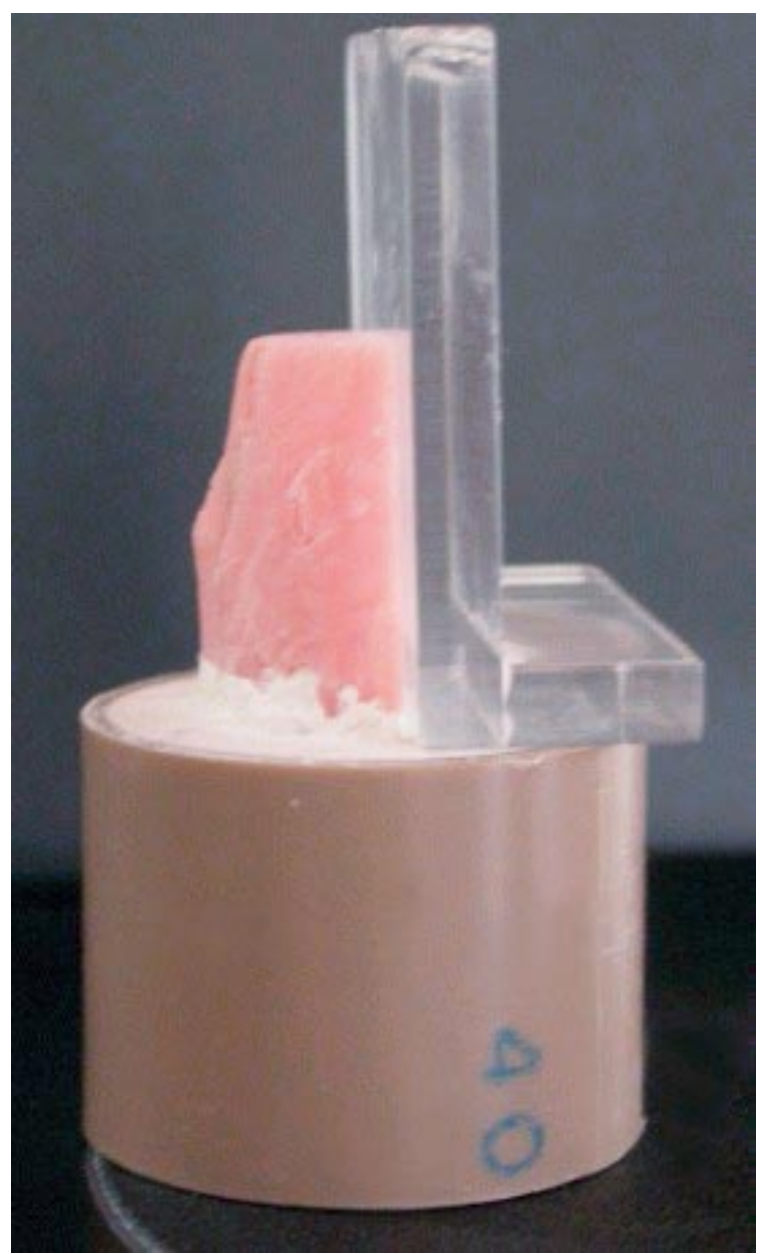

FIGURA 2 - Posicionamento do dente bovino já incluído na base, com auxílio de posicionador. 
suas faces vestibulares perpendiculares à base do troquel. Para isso utilizou-se um posicionador confeccionado exclusivamente para tal finalidade (Fig. 2).

Após a inclusão, foi realizado os procedimentos usuais de uma colagem, começando pela profilaxia com pedra-pômes e água por 10 segundos, utilizando-se micromotor de baixa rotação e taça de borracha. Em seguida, lavou-se pelo período de 10 segundos e posteriormente cada superfície foi seca com jatos de ar por 20 segundos. Uma vez realizada a profilaxia, lavagem e secagem foi feito o condicionamento com ácido ortofosfórico (37\%) por 20 segundos, seguido de lavagem e secagem por igual tempo. Dos 24 dentes utilizados no estudo, 12 foram colados com o compósito Concise 1929 (Grupo C) marca 3M do Brasil e 12 com o compósito Fill Magic Orthodontic (Grupo F) marca Vigodent, utilizando braquetes código 20.30.201 tipo standard da marca Morelli.

Os braquetes foram colados com o auxílio de posicionador (Fig. 3), sendo mantido pressionado por um determinado período, até que completasse a polimerização. Durante o período que estava sendo mantido em posição foram removidos os excessos de resina com o auxílio de uma sonda exploradora marca Duflex.

Os braquetes colados aos dentes foram removidos utilizando-se uma máquina Instron (Fig. 4), com velocidade de $1 \mathrm{~mm}$ por minuto para avaliação da resistência ao cisalhamento.

Após a remoção dos braquetes, cada superfície de esmalte foi classificada de acordo com os escores do IRA: 0 = nenhuma quantidade de material aderido ao dente; 1 = menos da metade do material aderido ao dente; 2 = mais da metade do material aderido ao dente e $3=$ todo o material aderido ao dente, inclusive a impressão da malha do braquete, segundo Artün e Bergland ${ }^{3}$.

\section{RESULTADOS E DISCUSSÃO}

Nesta pesquisa a resistência ao cisalhamento do grupo Concise (C) foi, em média,

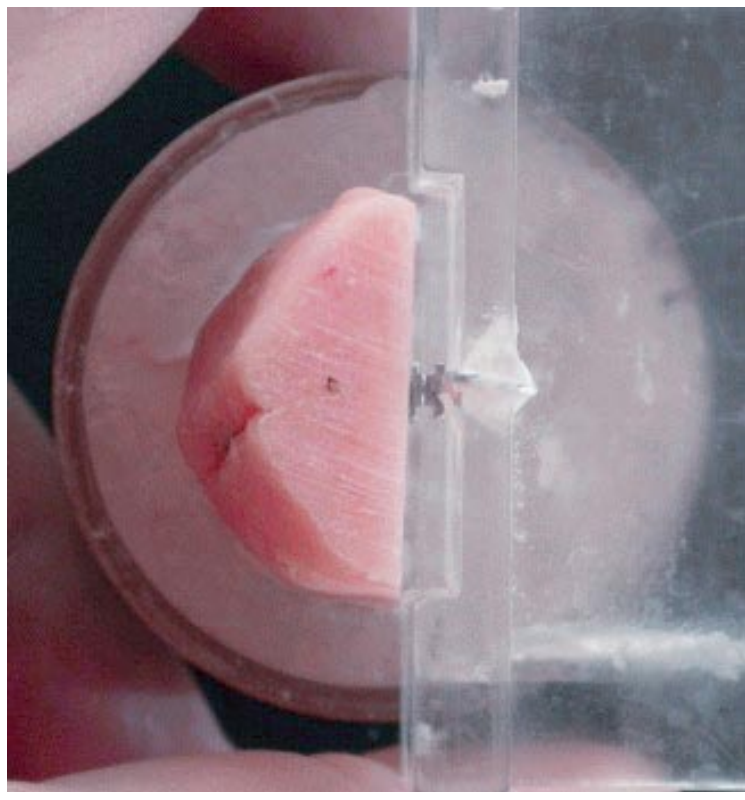

FIGURA 3 - Colagem dos braquetes com auxílio de posicionador, confeccionado para tal finalidade.

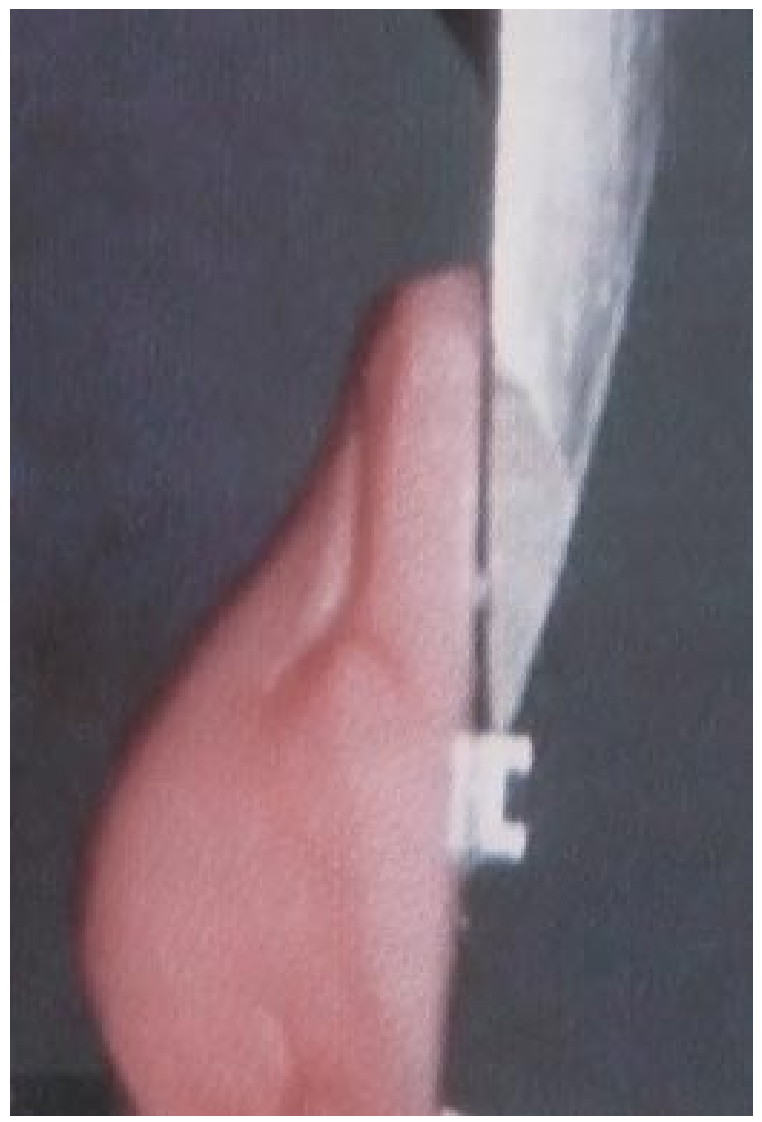

FIGURA 4 - Remoção do braquete com a máquina de ensaios de cisalhamento Instron. 


\begin{tabular}{ccccc}
\hline & resistência ao cisalhamento & \multicolumn{2}{c}{ IRA } \\
\hline materiais & Concise & Fill Magic & Concise & Fill Magic \\
médias & 55,01 & 58,69 & 1,60 & 2,26 \\
teste t & \multicolumn{2}{c}{0,291} & & \multicolumn{2}{c}{0,058} \\
\hline
\end{tabular}

Quadro 1 - Médias das resistências ao cisalhamento (em $\mathrm{KgF} / \mathrm{cm}^{2}$ ) e do IRA obtidas para os compósitos estudados e valor de significância do teste $t$.

$55,01 \mathrm{KgF} / \mathrm{cm}^{2}$ (desvio-padrão= 19,10 ) e do grupo Fill Magic (F) foi de $58,69 \mathrm{KgF} / \mathrm{cm}^{2}$ (desvio- padrão=17,70). O IRA dos grupos C e F foram, respectivamente, 1,60 e 2,26. Estes resultados sugerem que, em relação à resistência ao cisalhamento, não houve diferença estatística significante ao nível de $5 \%$ de probabilidade entre o grupo que utilizou o Concise e o que utilizou Fill Magic Orthodontic (Quadro 1). Resultados esses que corraboram com os encontrados por Araújo et al. ${ }^{2}$, Correr et al. ${ }^{10}$ e Santos et al. ${ }^{21}$, que testaram o Concise com outros materiais de colagem.

Essa constatação em relação ao IRA é de grande interesse para o ortodontista, que pode assim escolher materiais que apresentam respostas clínicas com quantidade maior de remanescente de adesivo na face dentária após a remoção do braquete, uma vez que pode proporcionar maior segurança, evitando as fraturas de esmalte e mantendo a integridade do dente. A remoção do remanescente resinoso na face dentária não consiste em um processo de difícil execução, uma vez que a remoção do adesivo das faces dentárias após a remoção do aparelho é uma rotina. Sendo assim, a escolha do material de colagem depende de uma avaliação criteriosa de suas propriedades clínicas.

Em relação ao IRA não houve diferença estatística significante entre as médias dos seus valores quando os dois grupos foram comparados, pois ambos apresentaram um maior número de fraturas na interface braquete/compósito (Quadro 1).

Reinolds e Fraunhofer ${ }^{18}$ relataram que para os valores de força de resistência à colagem suprirem as necessidades clínicas é necessária uma resistên- cia da colagem em torno de 60 a $80 \mathrm{KgF} / \mathrm{cm}^{2}$. No presente estudo, foram encontradas forças médias menores para os compósitos Concise e Fill Magic, quando comparadas aos valores relatados por Reinolds e Fraunhofer ${ }^{18}$, as quais apresentaram pequena diferença estatística entre si, embora estes valores de força sejam semelhantes aos achados por Myaki et al. ${ }^{14}$; Oliveira ${ }^{15}$ e Ward et al. ${ }^{24}$

No trabalho de Simplício ${ }^{23}$, que utilizou 60 fragmentos dentários, divididos em 3 grupos com 20 cada, no qual os acessórios foram colados com Concise ortodôntico (compósito pasta/pasta autopolimerizável), Rely-a-Bond (resina composta pasta única fotopolimerizável) e Fuji Ortho LC (cimento de ionômetro de vidro modificado por resina, com tripla presa), encontrou-se no teste de cisalhamento uma média de $21,45 \mathrm{KgF} / \mathrm{cm}^{2}$ e desvio-padrão de 5,47 para o grupo Concise, que mostrou-se superior aos demais materiais empre$\operatorname{gados}^{17}$.

\section{CONCLUSÃO}

Com base nos resultados obtidos, pode-se concluir que:

1) Quanto à resistência ao cisalhamento:

1.1) Os dois materiais testados apresentaram resultados semelhantes, não havendo diferenças estatisticamente significantes.

2) Quanto ao IRA:

2.1) Os dois materiais testados apresentaram resultados semelhantes, não havendo diferenças estatisticamente significantes.

3) Ambos os materiais satisfazem plenamente as necessidades clínicas para colagem de braquetes. 


\title{
A in vitro comparative study about shear bond strength and adhesive remnant index of the Concise and Fill Magic composites
}

\begin{abstract}
Aim: to compare the shear bond strength and adhesive remnant index (IRA) between the composites Concise and Fill Magic Orthodontic. Methods: the sample consisted of 24 cattle permanent low incisors divided in two groups. In the first group 12 brackets were bonded with the Concise composite and in the second group 12 with the Fill Magic Orthodontic composite. The tests of the shear and the evaluation of the ARI were developed with the whole sample. Results and Conclusion: there were not significant statistic differences between the composites in the two evaluated items.
\end{abstract}

Key words: Bonding. Shear bond strength. Debonding.

\section{REFERÊNCIAS}

1. ALEXANDRE, P. et al. Bond strength of three orthodontic adhesives. Am J Orthod, St. Louis, v. 79, n. 6, p. 653-660, June 1981.

2. ARAÚJO, M. T. S. et al. Contaminação de saliva humana na colagem de cimento de ionômero de vidro e de compósito em esmalte bovino: resistência ao cisalhamento. Rev Bras Odontol, Rio de janeiro, v. 55 , n. 1, p. 45-47, jan./fev. 1998

3. ARTÜN, J.; BERGLAND, S. Clinical trials with crystal growth conditioning as an alternative to acid-etch enamel pretreatment. Am J Orthod, St. Louis, v. 85, no. 4, p. 333-340, Apr.1984.

4. ARTÜN, J.; ZACHRISSON, B. U. Improving the handling properties of a composite resin for direct bonding. Am J Orthod, St. Louis, v. 81, no. 4, p. 269-276, Apr. 1982.

5. BUONOCORE, M. G. A simple method of increasing the adhesion of acrylic filling materials to enamel surface. J Dent Res, Chicago, v. 34, no. 6, p. 849-853, Dec.1955.

6. BUONOCORE, M. G. Retrospections on bonding. Dent Clin North Am, Philadelphia, v. 25, no. 2, p. 242-255, Apr. 1981.

7. CACCIAFESTA, V. et al. Effects of saliva and water contamination on the enamel shear bond strength a light-cured glass ionomer cement. Am J Orthod Dentofacial Orthop, St. Louis, v. 113 , no. 4, p. 402-407, Apr. 1998.

8. CHUNG, C. et al. Shear bond strength of a resin-reinforced glass ionomer cement. An in vitro comparative study. Am J Orthod Dentofacial Orthop, St. Louis, v. 115, no. 1, p. 52-54, Jan. 1999.

9. CORDEIRO, A. C.; BUSSADORI, S. K.; CAMARGO, M. C. F. Colagem de bráquetes com cimento de ionômero de vidro modificado por resina. Rev Paul Odontol, São Paulo, v. 21, n. 6, p. 10-15, nov./dez. 1999

10. CORRER SOBRINHO, L. et al. Avaliação da resistência ao cisaIhamento na colagem de bráquetes, utilizando diferentes materiais. Rev ABO Nac, Rio de janeiro, v. 9, n. 2, p. 157-162, jun./jul. 2001.

11. GORELIK, L. et al. Bonding metal brackets with a self-polymerizing sealant composite: a 12 month assessment. Am J Orthod, St. Louis, v. 75, no. 5, p. 542-553, May 1977.

12. GRANDO, P. R. et al. Colagem de backet ortodôntico com resina composta e com ionômero de vidro. J Bras Ortodon Ortop. Facial, Curitiba, v. 7, n. 38, p. 118-124, mar./abr. 2002.

13. GROSS, M. W. et al. Direct bonding to adlloy-treated amalgam. Am J Orthod Dentofacial Orthop, St. Louis, v. 112, no. 3, p. 252-258, Mar. 1997.

14. MYAKI, S. I. et al. Adesão de botões ortodônticos ao esmalte de dentes decíduos cimentados com resina composta ou ionômero de vidro modificado por resina. J Bras Ortodon Ortop Maxilar, Curitiba, v. 3, n. 14, p. 19-22, mar./abr. 1998.

15. OLIVEIRA, I. B. C. Avaliação laboratorial da força de adesão de diferentes materiais utilizados na colagem de braquetes metálicos ao esmalte dentário. 1999. Trabalho de Conclusão de Curso (Especialização)- Escola de Aperfeiçoamento Profissional da ABO, Curitiba, 1999

16. ORTENDAHL, T.; THILANDER, B. Use of glass-ionomer for bracket bonding-an ex vivo study evaluating a testing device for in vivo purposes. Eur J Orthod, London, v. 20, no. 2, p. 201-208, Fev. 1998.

17. PINZAN, C. R. M. et al. Estudo comparativo da resistência às forças de cisalhamento, de colagem de braquetes ortodônticos, testando dois tempos diferentes de condicionamento ácido, com e sem homogeneização prévia das pastas. Rev Dental Press Ortodon Ortop Facial, Maringá, v. 6, n. 6, p. 45-49, nov./dez. 2001.

18. REYNOLDS, I. R.; FRAUNHOFER, J. A. Direct bonding in orthodontics: a comparison off attachments. Br J Orthod, London, v. 4, n. 2, p. 65-69, 1976.

19. ROMANO, F. L.; RUELLAS, A. C. O. Estudo Comparativo in vitro da resistência ao cisalhamento da colagem e do índice de remanescente resinoso entre os compósitos concise e superbond.

Rev Dental Press Ortodon Ortop Facial, Maringá, v. 8, n. 1, p. 69-75, jan./fev. 2003.

20. SADLER, J. F. A survey of some commercial adhesives: their possible application in clinical orthodontics. Am J Orthod St. Louis, v. 44, no. 1, p. 65, Jan. 1958

21. SANTOS, P. C. F. et al. Colagem em ambiente úmido: avaliação da capacidade de resistencia à tração de bráquetes metálicos. Rev Dental Press Ortodon Ortop Facial, Maringá, v. 5, n. 6 p. 33-43, nov./dez. 2000.

22. SILVA FILHO, O. G. et al. Avaliação clínica da eficácia de um cimento de ionômero de vidro fotopolimerizável (Vitrebond) para a colagem direta de braquetes ortodônticos em nivelamento 4×2. Rev Dental Press Ortodon Ortop Facial, Maringá, v. 4 n. 1, p. 31-44, jan./ fev. 1999.

23. SIMPLÍCIO, H. M. Avaliação in vitro de materiais utilizados para colagem ortodôntica: potencial cariostático, resistência ao cisalhamento e padrão de colagem. 2000. Tese (Doutorado)-Faculdade de Odontologia de Araraquara, Universidade Estadual Paulista, Araraquara, 2000.

24. WARD, G. I. et al. Electrothermal bonding: shear bond strength of orthodontic brackets after two weeks. Br J Orthod, London, v. 25 , no. 2, p. 123-126, Fev. 1998

25. ZACHRISSON, B. U. Bonding in orthodontic. In: GRABER, T. M.; WAIN, B. F. Orthodontic current principle and technique. St. Louis: C. V. Mosby, 1985. p. 485-563.

\section{Endereço de correspondência}

Matheus Melo Pithon

Rua México 78

CEP: 45.020-390 - Recreio Vitória da Conquista / BA

E-mail: matheuspithon@bol.com.br 